E.L.U.A. 3, 1985-1986, págs. 113-128

\title{
IDEOLOGÍA DE LA «PUESTA EN TEXTO»EN «LA TÍA JULIA Y EL ESCRIBIDOR» DE MARIO VARGAS LLOSA
}

\author{
JACQUES SOUBEYROUX \\ (Universidad Paul Valéry. Montpellier)
}

En un artículo titulado «Pour une socio-critique ou variations sur un incipit", publicado en el número 1 de la revista Littérature, y que se presentaba como un verdadero manifiesto por una "socio-crítica" de los textos literarios, Claude Duchet definía el espacio nuevo en el cual pretendía fundar esa respectiva crítica como un espacio intermediario,

"entre-deux ouvert entre la sociologie de la création, à laquelle le nom de Lucien GOLDMANN demeure attaché, et la sociologie de la lecture, dont Bordeaux et Liège entre autres, onf fait leur spécialité". (Duchet, 1971: 6)

Y proponía unos objetos de estudio nuevos, casi nunca tenidos en cuenta por la crítica hasta entonces, y situados en la frontera del texto,

"zone indécise où se définissent les conditions, où se mêlent deux séries de codes: le code social, dans son aspect publicitaire, et les codes producteurs ou régulateurs du texte." (Duchet, 1971: 6)

En esta zona fronteriza, Duchet sitúa el título, la portada, las variantes, pero también la primera y la última frase de una novela. $Y$ para ilustrar sus propuestas teóricas, nos ofrecia a continuación, en la segunda parte del artículo, un análisis brillante de la frase inicial ( «el 
incipit») en Madame Bovary, como ejemplo de lo que el crítico llamaba "mise en texte" (Duchet, 1980), o sea de ese «processus actif d'appropriation de la réalité» (Duchet, 1971: 9), que permite crear en unas cuantas palabras las condiciones de existencia del texto por la constitución de un espacio novelesco, de unos personajes, de una ideología, de la literalidad del texto, a partir de los códigos socioculturales vigentes.

Como miembro del Centro de Estudios Sociocríticos de Montpellier (C.E.R.S.), que se ha ido desarrollando bajo la dirección de Edmond Cros paralelamente al de Paris VIII, animado por Duchet, a los quince años de publicación de este artículo, no puedo menos de expresar ante las propuestas de Claude Duchet y su aplicación un doble sentimiento de adhesión y rechazo, que requiere la explicitación de algunas nociones teóricas.

La perspectiva adoptada por Duchet para situar la "socio-critica» -ese espacio intermediario entre una sociología de la creación y una sociologia de la lectura- me parece ambigua y propia para crear un confusionismo peligroso. Entre los objetos analizados por Duchet, algunos que pertenecen al Ilamado «avant-texte" (el título, la portada) dependen exclusivamente de una sociología de la lectura, mientras otros, como la primera y la última frases de una novela, pertenecen verdaderamente al texto, y pueden ser tenidos en cuenta por una auténtica sociocrítica de la producción. La posición de Duchet que consiste en tratar de conciliar (¿mezclar, confundir?) dos perspectivas críticas fundamentalmente diferentes no me parece aceptable. La fórmula misma utilizada (ya citada) puede hacer creer que los códigos sociales sólo existen fuera del texto, al nivel de una sociología de la lectura (códigos publicitarios), cuando la segunda parte del artículo muestra que los signos sociales abundan dentro del texto mismo, desde la primera frase.

Para nosotros, una auténtica sociocrítica sólo puede situarse en el nivel de la producción, y en esta perspectiva no puede existir nada antes del texto (ningún "avant-texte») sino los discursos (las prácticas) sociales (lo que solemos llamar «interdiscursividad») y los intertextos (la intertextualidad) que se cruzan en ese espacio latente que llamamos "genotexto", y que son absorbidos y transformados por el texto (Cros, 1983: 108 ss.). Dejando de lado los efectos inducidos de las múltiples lecturas contradictorias de un texto, que corresponden a presupuestos metodológicos totalmente distintos, lo que nos interesa a través del análisis de las diferentes realizaciones textuales (consideradas como "fenotextos") es tratar de balizar el "genotexto", poniendo de manifiesto las formaciones ideológicas y discursivas que confluyen en él, y 
los intertextos que participan en la fase de producción, para proponer una lectura crítica del sistema ideológico textual.

$\mathrm{Si}$ no puedo menos de expresar profundas divergencias ante las propuestas teóricas de Claude Duchet que remiten a una sociología de la lectura, en cambio no dejaré de proclamar mi adhesión al modelo del análisis del «incipit» de Madame Bovary, que me parece en conformidad con los presupuestos sociocríticos antes definidos y que corresponden a una verdadera semiología de la ideología, ya que muestra perfectamente cómo los códigos sociales y socioculturales están presentes y pueden ser descifrados dentro del texto analizado.

Este tipo de lectura constituye una vía eficaz de aproximación a un texto narrativo, que permite marcar desde el estudio de la microsecuencia inicial, lugar privilegiado de la "puesta en texto", los principales discursos sociales y códigos culturales que se invierten en el texto novelesco. Es este método el que he tratado de seguir para estudiar la ideología de la upuesta en texto" en una novela de Mario Vargas Llosa, La tía Julia y el escribidor.

La tía Julia y el escribidor es la quinta novela de Vargas Llosa. Si el contexto histórico-social de referencia (la Lima del ochenio odriista) y el tema principal (la adolescencia como aprendizaje) la relacionan como algunas de las obras anteriores del autor (Los Jefes, La Ciudad y los Perros, Los Cachorros, Conversación en la Catedral). Su estructura narrativa es única. La bipolaridad anunciada por el título remite aquí a dos niveles narrativos:

- Los capítulos impares ( $y$ el capítulo $X X$ ) desarrollan la autobiografía novelada (prefiero hablar de “autoficción») de un joven de dieciocho años, llamado Varguitas por los demás protagonistas, pero que lleva el nombre y el apellido del autor, Mario Vargas;

- los capitulos pares forman nueve historias, que no tienen relación entre sí ni con el relato principal, narradas en tercera persona por un narrador anónimo, al que el lector identifica con Pedro Camacho, personaje del primer nivel de la narración. Estos capítulos pares constituyen así unos elementos de ruptura, a la vez al nivel narrativo y temático, parecido a las novelas interpoladas en obras clásicas como el Quijote o Guzmán de Alfarache. Uno de los principales problemas planteados por la novela será pues el de las relaciones que existen entre los dos niveles narrativos, o sea, tratar de buscar, a través de unas realizaciones textuales disímiles, las estructuras profundas del texto narrativo y su coherencia ideológica (si existe). Es lo que me propongo con el análisis de la microsecuencia inicial del capítulo primero, correspondiente a! primer nivel narrativo, y de la del capitulo segundo, principio de los radioteatros de Pedro Camacho. 
1. La "puesta en texto» en el capítulo $1 .^{\circ}$ de «La tía Julia y el escribidor»

No nos limitaremos, como hace Claude Duchet en sus dos artículos $(1971,1980)$ al «incipit» reducido en sentido estricto a la primera frase, sino que estudiaremos el conjunto del párrafo primero, como primera microsecuencia discursiva y narrativa de la novela.

En ese tiempo remoto, yo era muy joven y vivía con mis abuelos en una quinta de paredes blancas de la calle Ocharán, en Miraflores. Estudiaba en San Marcos, Derecho, creo, resignado a ganarme más tarde la vida con una profesión liberal, aunque, en el fondo, me hubiera gustado más llegar a ser un escritor. Tenía un trabajo de título pomposo, sueldo modesto, apropiaciones ilícitas y horario elástico: director de Informaciones de Radio Panamericana. Consistia en recortar las noticias interesantes que aparecian en los diarios y maquillarlas un poco para que se leyeran en los boletines. La redacción a mis órdenes era un muchacho de pelos engomados y amante de las catástrofes Ilamado Pascual. Había boletines cada hora, de un minuto, salvo los de mediodía y de las nueve, que eran de quince, pero nosotros preparábamos varios a la vez, de modo que yo andaba mucho en la calle, tomando cafecitos en la Colmena, alguna vez en clases, o en las oficinas de Radio Central, más animadas que las de mi trabajo.

Considerando este párrafo como «le lieu stratégique d'une mise en texte" (Duchet, 1980: 95), trataremos de ver cómo se desarrolla en él toda una retórica de la apertura caracterizada por la respuesta a las tres preguntas cardinales de cualquier relato: ¿quién? ¿dónde? ¿cuándo?, que abordaremos en el orden inverso.

\section{¿Cuándo?}

«En ese tiempo remoto, yo era muy joven...». El imperfecto, tiempo de la diégesis, corresponde a un pasado "remoto", que se opone a otro pasado más próximo («más tarde») y a un presente que surge de repente con el verbo "creo" de la segunda frase, y que representa el tiempo de la enunciación.

En las dos primeras frases encontramos una verdadera dimensión temporal, que programa ya el desarrollo de toda la historia: la expre- 
sión «llegar a ser" sugiere un proceso de transformación a partir del pasado remoto que anuncia la trayectoria temporal del protagonista que nos conducirá desde el pasado remoto de la historia al presente en que tienden a fundirse presente de la enunciación y presente de la acción, representado en el epílogo (capítulo $X X$ ) por la expresión: "ahora que he vuelto a vivir en el Perú» (Vargas Llosa, 1977: 432).

Subrayaré en seguida la importancia de este proyectarse hacia el futuro, que marca desde las primeras líneas toda la estructura temporal de la novela, y que corresponde, como he mostrado en otro artículo (Soubeyroux, 1980: 388), a la influencia determinante del proyecto de Varguitas («llegar a ser un escritor»), a través del modelo de la autobiografía de Sartre, Les Mots. Como un héroe sartriano, Varguitas se identifica totalmente con su proyecto, y toda su acción tiende hacia la realización de éste. En contra de lo que suele ocurrir en las autobiografías tradicionales, en que los acontecimientos van encadenados unos a otros, cronológicamente, por una relación de causalidad (el pasado explica el presente que a su vez explica el futuro), aquí es el porvenir soñado el que explica y organiza el presente, borrando casi totalmente el pasado.

\section{¿Dónde?}

Es de advertir primero el fuerte grado mimético del espacio novelesco que se va conformando aquí tomando muchos elementos en la realidad limeña: la calle Ocharán, Miraflores, San Marcos, Radio Panamericana, Radio Central, la Colmena. A través de esos lugares se van dibujando los dos principales escenarios de la acción en los capítulos impares: el barrio de Miraflores (lugar de residencia de Varguitas y de su familia) y el centro urbano (lugar profesional: Universidad, estaciones de radio).

Pero esos lugares no son neutrales (mera reproducción anecdótica de una realidad vivida), sino que están saturados de connotaciones sociales e ideológicas, que nos remiten a unos códigos exteriores y anteriores al texto. El lector que conoce un poco la realidad sociológica limeña (discurso social contemporáneo) o las obras anteriores de Vargas Llosa (intertextualidad) reconocerá inmediatamente en Miraflores un barrio burgués, pero la descripción de la casa familiar («una quinta de paredes blanicas") basta para informar al lector ignorante de todos estos contextos extratextuales: el texto aparece así como sobrecodificado en el nivel sociológico.

Los demás lugares citados (la Universidad de San Marcos, institu- 
ción educativa tradicional, y más precisamente, su facultad de Derecho; las dos estaciones de radio largamente descritas en el párrafo siguiente) corresponden a unas instancias de poder que identificaremos con Aparatos Ideológicos del Estado burgués (Althusser, 1976):

- Aparato Ideológico educativo ("clases"),

- Aparato Ideológico juridico («derecho»),

- Aparato Ideológico Informativo («Radio Panamericana, Radio Central»),

que se suman al Aparato Ideológico familiar presente desde la primera frase. El espacio novelesco construido en este primer párrafo aparece así como el espacio de la reproducción y defensa de los valores sociales de la burguesía.

A esos lugares, que corresponden a otras tantas instancias de poder, se oponen otros dos lugares, la calle y la Colmena, que aparecen como dos refugios, pertenecientes a un espacio de evasión, de búsqueda de la libertad individual. Así se va esbozando, desde este primer párrafo, la estructura espacial de la novela caracterizada por la oposición entre unos espacios de poder (el centro, los barrios burgueses) y unos espacios de (búsqueda de la) libertad (los barrios periféricos, los lugares cerrados) (Soubeyroux, 1982a), estructura espacial que refleja el conflicto entre un individuo (Varguitas, o el protagonista principal de los capítulos pares) y la sociedad burguesa en que vive.

\section{¿Quién?}

Lo esencial de este primer párrafo consiste, sin embargo, en la creación de un personaje, que será el héroe de los capítulos impares, y que desde la primera línea dice "yo". Este actor aparece como un adolescente miembro de una familia burguesa antigua ( "los abuelos») en que impera la ley de la reproducción de los valores de su clase a través del modelo familiar y universitario: la «profesión liberal» aparece como la carrera obligada que asegura a la vez el porvenir económico y la pertenencia a la clase social. Desde las dos primeras frases, el yo aparece así inmerso en una realidad social y definido a partir de los valores ideológicos propios de la clase burguesa.

Sin embargo, desde la primera línea ("yo era muy joven») aparece afirmada en el texto la fundamental dualidad de ese yo, que remite por una parte al actor que protagoniza la historia en el pasado remoto, y por otra parte, al narrador en el presente de la enunciación. Estamos aquí ante uno de los problemas esenciales de cualquier autobiografía: la ineludible alteridad entre el sujeto del enunciado y el sujeto de la 
enunciación, oculta bajo la unidad aparente del yo, que no es más que una ilusión creada por el relato. El verbo «creo» de la segunda parte, aparente duda o fallo de memoria del narrador, sirve para marcar la distancia irónica entre el adolescente inexperimentado de la historia, y el narrador del relato, imagen del autor consagrado.

Pero más allá de esa alteridad inherente a toda autobiografia, encontramos otra alteridad, todavía más subrayada por el texto, en la personalidad del mismo actor. Varguitas aparece como un personaje doble: el adolescente que vive con sus abuelos y ha aceptado por fuerza ( «resignado») una carrera (el derecho) y un proyecto de porvenir ( «ganarme más tarde la vida con una profesión liberal») impuesto por la familia, que se oponen a su vocación personal, secreta, pero afirmada de modo explícito desde las primeras líneas de la novela, y nacida antes del principio de la historia ( «llegar a ser un escritor»). Lo que caracteriza al actor desde el «incipit» de la novela es, pues, el conflicto entre un proyecto auténtico, que corresponde a unos valores ideales (la literatura), y una forma de vida inauténtica, que corresponde a la reproducción de los valores burgueses. Este conflicto, marcado en el texto por la conjunción adversativa "aunque» y por la oposición entre las expresiones "resignado a" y "me hubiera gustado más», es el germen de la lucha que va a estallar entre Varguitas y la familia (la sociedad) y anuncia toda la acción de los capítulos impares.

La dualidad del personaje y la inautenticidad de su situación, puestas de relieve en las dos primeras frases, están confirmadas en el sistema semiótico de las frases siguientes por la ocurrencia de términos como «ilícitas», «maquillar», la conjunción "pero», y por una serie de oposiciones:

«título pomposo" «había boletines cada hora, de un minuto»
VS. «sueldo modesto»

VS. «horario elástico» «nosotros preparábamos varios a la vez».

Inautenticidad en el trabajo: la función de «director de Informaciones" sólo consiste en "recortar» y "maquillar» artículos publicados en la prensa, o sea una actividad que se sitúa en los antípodas de su vocación de escritor.

A la primera oposición entre las fuerzas burguesas («mis abuelos», "San Marcos», "profesión liberal») y el yo auténtico del actor sucede aquí una nueva oposición entre título oficial («director de Informaciones»), expresión de la "falsa" jerarquía social, y vida auténtica del actor. Con la complicidad de Pascual, asistimos a la formación de un grupo ( «nosotros») que se opone a los valores burgueses, y logra bur- 
larse de las exigencias del oficio para escapar a la calle o a los cafés. Este grupo anuncia el de los adolescentes rebeldes o de los marginales, en lucha contra los valores dominantes de la sociedad, que encontraremos en los capítulos pares de la novela (Soubeyroux, 1982a).

A la apariencia de un horario rígido, se opone la gran libertad lograda por el protagonista e invertida en unas distracciones («andar por la calle», «tomar cafecitos») que corresponden a un tiempo vacio equivalente a unas posibilidades de acción (podria hablarse de una matriz de espera) que serán utilizadas por el «embrague» de la historia, con la aparición simultánea de la tía Julia y de Pedro Camacho.

Así, desde las primeras frases del texto, están creadas las condiciones para el desarrollo futuro de la acción del protagonista, que adopta enseguida los rasgos propios al actante sujeto definido como adolescente enajenado en busca de un orden nuevo, que corresponde al modelo de varias novelas existencialistas, por ejemplo Les chemins de la Liberté de Jean-Paul Sartre. Como en estas novelas sartrianas, el conflicto ideológico, ya legible directamente en el «incipit», consiste en la problematización de los valores burgueses (la familia, el dinero, la profesión, la universidad, el derecho, los medios de comunicación) opuestos a unos valores personales e ideales (la libertad individual, la literatura) a través de una estructura dialéctica de ocultación/desocultación, cuyo eje es la permanente ambigüedad del yo que se expresa en el relato a lo largo de los capítulos impares, y que será según los casos el yo del actor enajenado ( «resignado»), el yo auténtico (secreto) del actor Varguitas o el yo del narrador que tiende a confundirse (pero, ¿hasta qué punto?) en el relato autobiográfico (más exactamente autoficticio) con el yo del autor, Mario Vargas Llosa.

\section{La «puesta en texto» en el capítulo segundo de «La tía Julia y el escribidor»}

\section{II}

Era una de esas soleadas mañanas de la primavera limeña, en que los geranios amanecen más arrebatados, las rosas más fragantes y las buganvilias más crespas, cuando un famoso galeno de la ciudad, el doctor Alberto de Quinteros -frente ancha, nariz aguileña, mirada penetrante, rectitud y bondad en el espírituabrió los ojos y se desperezó en su espaciosa residencia de San Isidro. Vio, a través de los visillos, el sol dorando el césped del cuidado jardín que encarcelaban vallas de crotos, la limpieza del cielo, la alegría de las flores, y sintió esa sensación bienhechora 
que dan ocho horas de sueño reparador y la conciencia tranquila.

Era sábado y, a menos de alguna complicación de último momento con la señora de los trillizos, no iría a la clínica y podria dedicar la mañana a hacer un poco de ejercicio y a tomar una sauna antes del matrimonio de Elianita. Su esposa y su hija se hallaban en Europa, cultivando su espíritu y renovando su vestuario, y no regresarían antes de un mes. Otro, con sus medios de fortuna y su apostura - sus cabellos nevados en las sienes y su porte distinguido, así como su elegancia de maneras, despertaban miradas de codicia incluso en señoras incorruptibles-, hubiera aprovechado la momentánea soltería para echar algunas canas al aire. Pero Alberto de Quinteros era un hombre al que ni el juego, ni las faldas ni el alcohol atraían más de lo debido, y entre sus conocidos -que eran legión- circulaba este apotegma: «Sus vicios son la ciencia, su familia y la gimnasia».

Al analizar los dos párrafos iniciales del capítulo segundo de la novela, que representan la primera microsecuencia del otro nivel narrativo, el de los radioteatros de Pedro Camacho, nuestra intención es tratar de mostrar hasta qué punto y a través de qué procedimientos retóricos se da en él un planteamiento ideológico parecido (o no) al que hemos puesto de relieve en el capítulo primero, y que corresponde, bajo la heterogeneidad de las realizaciones textuales, a la unidad ideológica del texto novelesco.

La primera frase de este capítulo segundo aparece en seguida como un «incipit» realista de carácter descriptivo, que toma numerosos elementos en el mundo real. Como en el capítulo primero, el texto utiliza el conocimiento que el lector tendrá de la sociología de los barrios limeños: el nombre de "San Isidro" opera como signo social, que clasifica a sus habitantes en la alta burguesía (la oligarquía) de la capital.

El deíctico «esas» («una de esas soleadas mañanas de la primavera limeña") constituye una llamada directa a este conocimiento previo del lector. Pero, aunque el lector no tenga ningún conocimiento de la realidad limeña, el apellido del personaje, su profesión y la larga descripción del lugar de acción (las flores que adornan los jardines de la "espaciosa residencia») forman un conjunto de signos sociales que no pueden dejar duda alguna sobre la clase social y la riqueza del protagonista.

Aunque la «espaciosa residencia de San Isidro" puede sugerir un nivel de riqueza superior al de la "quinta de paredes blancas de la calle Ocharán, en Miraflores», del capítulo primero (pero también podria considerarse la una como proyección hiperbólica de la otra), los dos 
textos aluden a un mismo grupo social, el de las profesiones liberales (abogado en el capítulo primero, médico en el segundo) e insisten en los mismos valores burgueses: el dinero y la familia. La riqueza del doctor Quinteros está connotada, como hemos visto, por todos los signos de la primera frase. Y el párrafo segundo va a confirmarla con las alusiones a la clínica y al viaje de su esposa y su hija por Europa. La "espaciosa residencia de San Isidro»es, ante todo, en el texto, el lugar de la vida familiar: el párrafo segundo del texto insistirá en la importancia de la familia con el anuncio del matrimonio de la prima del médico, Elianita, con la alusión a su esposa y su hija, y sobre todo con el "apotegma» que «circulaba entre sus conocidos": «Sus vicios son la ciencia, su familia y la gimnasia».

En estos dos párrafos, el protagonista está presentado como un hombre solitario, aislado de los demás y del mundo exterior. Esta noción de aislamiento está marcada en el sistema semiótico del texto por palabras como «visillos», «encarcelaban», «vallas», etc. El párrafo segundo insiste en la total independencia y libertad de acción del personaje, sugiriendo algunas posibilidades («la señora de los trillizos», "echar algunas canas al aire») que son inmediatamente rechazadas, para insistir en la seriedad ejemplar del doctor, y dejarle mucho tiempo libre que será ocupado en actividades deportivas características de un estilo de vida burgués (gimnasia, sauna). Como el protagonista del capítulo primero, el doctor Quinteros dispone, pues, de unas posibilidades de acción inexplotadas, que constituyen otra «matriz de espera» que corresponderá al desarrollo de la historia.

Pero la diferencia esencial entre los dos personajes es su relación con el mundo que los rodea: mientras Varguitas se presenta como un héroe enajenado, en conflicto con la sociedad burguesa en que vive, Alberto de Quinteros aparece como uno de los representantes típicos de esta sociedad, totalmente de acuerdo con los valores dominantes, «perfectamente reconciliado con su mundo», diria Vargas Llosa. La segunda frase del texto en particular, con expresiones como «sueño reparador», «conciencia tranquila», insiste en el carácter no problemático del protagonista.

Entre el capítulo primero y el capítulo segundo, asistimos pues a una inversión de perspectivas: frente al adolescente «resignado», víctima de la sociedad burguesa que protagoniza el capítulo primero, tenemos aquí un burgués de cincuenta años, que encarna los valores de esta sociedad y goza totalmente de ellos. La problematización de los valores burgueses en el capitulo segundo sólo vendrá al final, después de una inversión estructural (Soubeyroux, 1982b: 110-111), y será provocada por dos adolescentes, Elianita y Richard. 
Sin embargo, la eliminación de todo posible conflicto en el nivel de la historia en los dos párrafos iniciales del capítulo segundo no significa que la ideología burguesa desarrollada a través del retrato del doctor Quinteros no está criticada desde el principio. Pero esta crítica no sigue las mismas vias que en el capítulo primero: en vez de inscribirse en la acción misma, está escrita en el nivel formal e intertextual de la «puesta en texto".

La primera fase, que constituye aparentemente un «incipit» realista por las referencias al contexto extratextual, multiplica los procedimientos tópicos de la "puesta en texto", dando amplia respuesta a las tres preguntas cardinales del relato: ¿cuándo? ¿dónde y ¿quién?

\section{¿Cuándo?}

Desde el principio, el texto precisa la hora aproximada y la estación del año en que se desarrolla la escena: «una de esas soleadas mañanas de la primavera limeña». Pero lo que podía aparecer a primera vista como una situación concreta no es más que una acumulación de tópicos de la apertura: el texto se abre en el momento del despertar del personaje («abrió los ojos»), que corresponde al principio del día (la mañana) y al despertar de la naturaleza (la primavera). Además, el cuadro evocado, muy idealizado («el sol dorando el césped, la limpieza del cielo") parece más próximo al clima de California, de la Costa Azul o de la Costa del Sol que no a la humedad y al cielo plomizo de Lima en la primavera. El carácter realista del «incipit" se desvanece asi detrás de 10 inauténtico del enunciado, y la comunicación con el narratario, instaurada por el deíctico «esas", no se realiza al nivel de un supuesto conocimiento de la realidad, sino al nivel intertextual de la parodia del «incipit» tradicional.

Recordemos el «incipit» de la novela de Georges Ohnet, Le Maitre de Forges, considerado por Claude Duchet como un enunciado canónico del «incipit» realista (Duchet, 1980: 102):

«Par une claire journée du mois d'octobre 1880, un jeune homme vêtu d'un élégant costume de chasse était assis à la lisière d'un de ces beaux bois de chênes qui couvrent de leur ombre fraîche les premières pentes du Jura".

Es este enunciado canónico el que sirve de modelo, varias veces transformado, al aprendiz de escritor de Camus en La Peste, quien escribe: 
«Par une belle matinée du mois de mai, une élégante parcourait, sur une superbe jument alezane, les allées fleuries du Bois de Boulogne.»

Podemos considerar que el «incipit» del capítulo segundo de La tia Julia y el escribidor se sitúa en la misma línea, y no es más que otra transformación (otra caricatura) del mismo enunciado canónico, utilizado por muchísimos textos paraliterarios (folletines, melodramas, etc.), que tanto gustan a Vargas Llosa, y que constituyen uno de los elementos básicos de la novela (Soubeyroux, 1982b).

\section{¿Dónde?}

Hemos visto que la primera frase del capítulo contiene varios signos referenciales que nos remiten al contexto social limeño. Pero la larga descripción acumula los elementos de decorado (los geranios, las rosas, las buganvilias, el césped, el cuidado jardín, las vallas de crotos, las flores) que sirven para constituir lo que Duchet Ilama un «lieu milieu» o «lieu atmosphère» (Duchet, 1980: 98) y crean un ambiente idealizado de belleza y alegría, en perfecta armonía con la riqueza y despreocupación del protagonista. La inautenticidad del enunciado estalla aquí en la acumulación de los adjetivos: no menos de diez en la primera frase, o sea mucho más que en el texto de Georges Ohnet, donde sólo, por decirlo así, se encontraban seis. Además de esta adjetivación, tan lujuriante como la vegetación, la repetición del comparativo («más arrebatados..., más fragantes..., más crespas») constituye. la primera característica del estilo rebuscado de los radioteatros de Pedro Camacho, parodia del estilo paraliterario.

\section{¿Quién?}

El «incipit» del capítulo segundo de La tía Julia y el escribidor no se contenta con introducir al protagonista: agota de una vez la suma de las preguntas que el lector podria hacerse sobre su identidad dándonos su nombre y apellido, su profesión, su retrato físico y moral, además de su lugar de residencia. Estamos aquí ante otro tópico de la «puesta en texto» que también pertenece a la caricatura del «incipit» realista.

La profesión elegida, expresada por la palabra rebuscada "galeno», que confirma el carácter paródico e inauténtico del enunciado, no vale sólo como característica social homóloga a la de la familia de Varguitas («profesión liberal»): es también un tópico de la paraliteratura, en que abundan los héroes pertenecientes a las profesiones médicas. Después 
de una encuesta llevada a cabo en Francia algunos años antes de su publicación de la novela de Vargas Llosa, Evelyne Sullerot afirmaba que el héroe preferido de una mayoría de lectores de fotonovelas era médico, o mejor aún cirujano, por representar éste a la vez un cientifico, un artista y un apóstol (Sullerot, 1970: 131).

El apellido del personaje, Alberto de Quinteros, que sugiere su ascendencia aristocrática, nos sitúa también en una tradición paraliteraria, ilustrada en particular por las novelas de Guy des Cars.

En cuanto al retrato del protagonista ( sfrente ancha, nariz aguileña, mirada penetrante, rectitud y bondad en el espiritu»), funciona en la novela como un signo que se repetirá en los nueve capítulos pares (hasta intercambiarse graciosamente los adjetivos en el capitulo XVIII), constituyendo con la edad (la cincuentena) la etiqueta distintiva del héroe en cada uno de ellos.

El párrafo segundo insiste en la «apostura» física del doctor («sus cabellos nevados en las sienes y su porte distinguido") y en su elegancia, que hacen de él un prototipo del seductor tan frecuente en todos los textos paraliterarios.

El análisis de los dos primeros párrafos del capítulo segundo de La tía Julia y el escribidor muestra que, antes de hacerse a través de la acción misma de la historia narrada (lo que ocurrirá en la segunda parte del capitulo con el descubrimiento del amor incestuoso entre Elianita y su hermano Richard), la contestación de la ideologia burguesa se realiza desde el «incipit» y las primeras líneas del capitulo por una parodia de todos los tópicos y procedimientos usuales de la paraliteratura, que vacía el enunciado de su contenido aparentemente realista. Utilizando una fórmula de Claude Duchet, podemos decir que «la critique de l'idéologie se fait dans l'idéologie d'un style, lui-même surdéterminé par l'ideologie qu'il conteste.» (Duchet, 1971: 14).

\section{Conclusión}

La lectura propuesta de los párrafos iniciales del capítulo primero y del capítulo segundo de La tía Julia y el escribidor, como lugar privilegiado de la "puesta en texto" de los dos niveles de la obra, ha tratado de estar atenta a la presencia de todos los signos sociales en el texto, pero también a la utilización de los códigos culturales y de los diferentes intertextos. Los resultados expuestos permiten ya balizar útilmente el "genotexto" de la novela:

- en el nivel interdiscursivo, el análisis hace patente la presencia recurrente de unos signos representativos de la ideología burguesa, en 
particular a través de varios Aparatos Ideológicos de Estado, entre los cuales dominan el Aparato familiar y el Aparato informativo. Pero este discurso burgués está sin cesar problematizado, en nombre de unos valores ideales (la libertad, la literatura) asumidos por un actor (Varguitas en el capítulo primero y en los demás capítulos impares) o directamente por la instancia narrativa en su parodia de la paraliteratura (capítulo segundo y demás capítulos pares);

- el nivel interdiscursivo está, pues, estrechamente vinculado al nivel intertextual, en el cual hemos podido advertir ya la presencia de tres intertextos importantes: la autobiografía sartriana (Les Mots) de donde procede toda la estructura temporal de los capítulos impares, dominada por la noción de proyecto; las novelas existencialistas, que proporcionan el prototipo del actante enajenado, adolescente en rebelión contra la sociedad burguesa, a partir del cual se organiza en gran parte el personaje de Varguitas; la paraliteratura como «arché-texte de genre», según una noción propuesta por Laurent Jenny (Jenny, 1976: 264), que me parece constituir el intertexto esencial de la obra, que influye a la vez en los capitulos pares y en los capítulos impares.

Hemos visto cómo, desde el «incipit» del capítulo segundo, el texto vargasllosiano se construye absorbiendo y parodiando los procedimientos y la retórica paraliteraria. En los capítulos impares, la paraliteratura está presente también con una verdadera puesta al desnudo de las fases de producción, difusión y recepción de los radioteatros de Pedro Camacho. Para los propietarios de Radio Central, los Genaros (calificados por Camacho de «mercaderes» y por el narrador de «empresario progresista»), los radioteatros no son más que una mercancía que se compra al kilo a un producto extranjero (Vargas Llosa, 1977: 15), y no tienen más valor que el que les atribuyen las fluctuaciones de la sintonía, de que dependen las tarifas publicitarias. El éxito de los radioteatros, producidos por Camacho como «sartas de salchichas» (Vargas Llosa, 1977: 158) y protagonizados por el mismo Camacho, se opone a los fracasos del aprendiz de escritor Varguitas, que trata de cultivar la literatura auténtica. Volvemos a encontrar asi una problemática (ya presente desde el principio del capítulo inicial de la novela con la crítica de las comunicaciones de masa y con el sueño de Varguitas de «llegar a ser un escritor») que ha sido desarrollada muchísimas veces por Vargas Llosa en artículos y conferencias (Vargas Llosa, 1983: 89 y 132): la del «analfabetismo cultural» de la sociedad peruana y de la trágica situación del escritor en ella. La paraliteratura (aquí los radioteatros) representa una producción destinada al consumo popular y sometida a clos mecanismos trituradores de la oferta y la demanda de la sociedad industrial», denunciados por Vargas Llosa en La Orgía per- 
petua (Vargas Llosa, 1975: 268-269). Todo eso tiende a mostrar que la paraliteratura es aquí mucho más que un simple intertexto: es una verdadera estructura de mediación entre la sociedad limeña burguesa neo-capitalista del ochenio odriista y el texto novelesco; a través de la temática y de los procedimientos de los radioteatros, el autor refleja, deformándolos por la parodia, la hipérbole, la caricatura, los problemas esenciales de esta sociedad burguesa y problematiza sus valores dominantes.

La lectura sociocrítica de las dos microsecuencias iniciales de los capítulos primero y segundo de la novela, atenta a la vez a las huellas de los discursos sociales (eje de la interdiscursividad) y a la resurgencia de los códigos culturales (eje de la intertextualidad) permite sugerir toda la riqueza de una obra considerada como menor por la critica, y muestra la fusión estrecha que existe en ella entre la historia y los procedimientos retóricos (entre fondo y forma) que participan de modo complementario en la crítica de la ideología burguesa.

Esta lectura podrá parecer a veces demasiado monosémica y un poco sencilla en el nivel ideológico. Es que las líneas iniciales que hemos estudiado sólo presentan las premisas del conflicto ideológico entre el actor principal Varguitas, doble del autor, y la sociedad burguesa, conflicto que se desarrolla a lo largo de la obra, y que no aparece claramente resuelto al final. En efecto, mientras toda la novela parecía abogar por una condena de los valores sociales burgueses en nombre de la libertad y de la literatura, el capítulo XX (que tiene la función de un verdadero epílogo) trata de conciliar de modo poco convincente los dos sistemas ideológicos antes opuestos: para justificar el regreso de Varguitas, que se ha convertido en Europa en un escritor consagrado, a la sociedad limeña de la que habia huido doce años antes, el texto pretende mostrarnos que esta sociedad limeña ha cambiado mucho. Pero el segundo casamiento de Varguitas (religioso esta vez) con su prima Patricia lo reintegra en su familia y en el sistema de valores contra el que no había cesado de luchar, y el lector puede preguntarse si es verdaderamente la sociedad la que ha cambiado o el personaje, doble del autor. Toda la novela es finalmente la historia del conflicto entre el autor y la sociedad limeña, y puede leerse como un intento de justificación de su salida a Europa y de su regreso al Perú. Pero esa interpretación global de la obra exigiria el análisis de muchas otras páginas de la obra, y va más allá de lo que se proponía mostrar este artículo. 


\section{Referencias bibliográficas}

Althusser, Louis (1973), “ldéologie et Appareils Idéologiques d'État», Positions, Paris, Editions Sociales.

Cros, Edmond (1983), Théorie et pratique sociocritiques, Montpellier, C.E.R.S., Université Paul Valéry.

Duchet, Claude (1971), «Pour une sociocritique ou variations sur un incipit», Littérature, $\mathrm{n} .^{\circ} 1$, pp. 5-14.

- (1980), «ldéologie de la mise en texte», La Pensée, n. ${ }^{\circ} 215$, pp. 95-107.

Jenny, Laurent (1976), “La stratégie de la forme», Poétique, n. ${ }^{\circ} 29$, pp. 257-267.

Soubeyroux, Jacques (1980), «El narrador y sus dobles (hacia una interpretación del universo narrativo de La tía Julia y el escribidor de Vargas Llosa", Hommage au Professeur J. L. Flecniakoska, Montpellier, Université P. Valéry, t. II, pp. 383-402.

- (1982a), «Espace vécu et structures textuelles: Lima dans La tia Julia y el escribidor de Vargas Llosa», Imprévue, n. ${ }^{\circ}$ 2, pp. 87109.

- (1982b), "Littérature et paralittérature dans La Tía Julia y el escribidor", Vargas Llosa, Co-textes n. 4 , Montpellier, C.E.R.S., pp. 101-135.

Sullerot, Evelyne (1970), «Les photoromans», Entretiens sur la paralittérature, Colloque de Cerisy, Paris, Plon, p. 119-141.

Vargas Llosa, Mario (1975), La Orgia perpetua: Flaubert y Madame Bovary, Barcelona, Seix Barral.

- (1977), La tía Julia y el escribidor, Barcelona, Seix Barral.

- (1983), Contra viento y marea (1962-1982), Barcelona, Seix Barral. 